\title{
Advances in the Inhibitors of Janus Kinase
}

\section{Jun-Jie J Jiang', Xiao-Ying Wang', Yv Zhang ${ }^{2}$, Yi Jin ${ }^{1 *}$ and Jun Lin ${ }^{1 *}$}

${ }^{1}$ Key Laboratory of Medicinal Chemistry for Natural Resource (Yunnan University), Ministry Education, School of Chemical Science and Technology, Yunnan University, Kunming, 650091, P. R. China

${ }^{2}$ State Key Laboratory of Drug Research, Shanghai Institute of Materia Medica, Shanghai Institutes for Biological Sciences, Chinese Academy of Sciences, 201203, China

\begin{abstract}
The janus kinases (JAKs) comprise an important class of non-receptor protein tyrosine kinases. Cytokine and receptor binding can cause the activation of JAKs, and then activate the "signal transcripts and transcriptional activator", making JAK enter the nucleus to induce target gene expression. JAKs regulate inflammatory diseases, bone marrow hyperplasia and a variety of malignant tumours. Using JAKs inhibitors as the therapy for organ transplantation and immune disease has a very important significance. In recent years, many of the JAKs inhibitors have been developed one after another. Pan JAKs inhibitor and selective JAK2 or JAK3 inhibitor research progress are reviewed in this article.
\end{abstract}

Keywords: Janus kinase; Inhibitors; Anti-tumour; Antiinflammatory

\section{Introduction}

In recent years, for the lesion site, studies at the molecular level of drugs targeting protein molecules has been a major research method for exploiting new, high-efficiency drugs with low toxicity [1]. Janus kinases (JAKs) are important signal transducers of many cytokines, growth factors and interferon. In recent years, it has been found in cancer cells and cells transfected with oncogenes that there would be a significant enhancement in the expression of JAKs. Also, the expression of JAKs has a close relationship with inflammation and autoimmune diseases and immune rejection of the allograft following formation [2]. With the research development of JAKs, a large number of safe, high-efficiency and highly selective JAKs inhibitors have been designed, which can selectively block JAKs signal transduction systems. According to clinical trial results, JAKs have high selectivity, high bioavailability, therapeutic activity and are well tolerated, which makes the treatment of diseases using JAKs possible [3]

\section{The Structure and Function}

Compared to other tyrosine kinases, JAKs are a family of nonreceptor tyrosine kinases that are relatively large molecules (120$130 \mathrm{kDa}$ ). There are four family members of JAKs: JAK1, JAK2, JAK3, and TYK2. JAK1, JAK2 and TYK2 exist in various tissues and cells (Figure 1), while JAK3 only exists in the marrow and lymphatic system. The four JAKs possess seven different domains (Figure 1). The JAK homology region (JH1) is the kinase domain and the more proximal region is $\mathrm{JH} 2$, which is functionally equivalent to negative regulatory factors and is an inactive pseudokinase domain. In addition, there are also five kinds of N-terminal domains (JH3-JH7), mainly involved in the binding of receptors. In the JAK family, there is a high degree of homology and highly homologous binding to the catalytic domain can be observed. One of the functional structures is $\mathrm{JH} 1$ and $\mathrm{JH} 2$, with $\mathrm{JH} 1$ being the active catalytic region. Though JH2 does not decide the catalytic action, it is considered to play an important role. When stabilising and confirming JAK, JH3 and JH4 domains (SH2) are considered to play a primarily structural role, while JH5-JH7 domains (FERM domain) have shown interactions with the extracellular domain of the cytokine receptors directly and JH1 [4].

JAKs transmit extracellular stimuli through signals that are generated by the relevant receptors [5]. Receptors and/or JAKs selectively activate signal transduction and signal transducer and activator of transcription (STAT) proteins by phosphorylation sites near the $\mathrm{SH} 2$ domain [6].

JAK1 signal conduction mainly relies on a variety of cytokines, making them phosphorylated and increasing their ability to activate downstream signalling molecules. A variety of cytokines, such as INF-A, INF-B, INF-Y, IL-6, and IL-7, can be applied to JAK proteins to make them phosphorylated. By activating its downstream STAT signalling molecules, the JAKs/STATs pathway continues to activate and initiate the proliferation and differentiation of the haematopoietic system without exception. With in-depth studies of the JAK-STAT pathway, the role of JAK1 in solid tumours has gradually been taken seriously. At present, many studies have shown that JAK1 plays an important role in the treatment of breast cancer, prostate cancer, head and neck cancer, kidney cancer, uterine sarcoma and malignant melanoma $[7,8]$.

JAK2 is widely distributed in the cytoplasm of cells that are involved in signal transduction of the haematopoietic and immune systems. It is critical to generate red blood cells and activate immune cells. Epidermal growth factor, platelet-derived serum factor, and colony stimulating factor are all involved in the signal transduction pathways associated with JAK2, which induce cellular proliferation, differentiation and apoptosis. The JAK2 and STAT families contain several members constituting multiple signal transduction pathways, such as JAK2/STAT3, JAK2/STST5. The JAKs/STATs pathway induces receptor dimerisation through ligands and receptors on the cell surface bind to each other. Receptor dimerisation causes phosphorylation of each other, thereby activating JAK. The activated, phosphorylated JAK receptors form the binding sites on the receptor and phosphorylate STAT. Homologous or heterologous dimers are formed by the activated STAT moving to the nucleus, and combining with gene expression promoters to regulate gene expression. Through the signalling pathway, JAK2 is involved in the development, differentiation and apoptosis

*Corresponding authors: $\mathrm{Yi}$ Jin and Jun Lin, Key Laboratory of Medicinal Chemistry for Natural Resource (Yunnan University), Ministry Education, Schoo of Chemical Science and Technology, Yunnan University, Kunming, 650091, P. R. China, Tel/Fax: +86 871503 3215; E-mail: jinyi@ynu.edu.cn, linjun@ynu.edu.cn

Received July 18, 2014; Accepted July 29, 2014; Published July 31, 2014

Citation: Jiang JJJ, Wang XY, Zhang Y, Jin Y, Lin J (2014) Advances in the Inhibitors of Janus Kinase. Med chem 4: 540-548. doi:10.4172/2161-0444.1000192

Copyright: $\odot 2014$ Jiang JJJ, et al. This is an open-access article distributed under the terms of the Creative Commons Attribution License, which permits unrestricted use, distribution, and reproduction in any medium, provided the original author and source are credited. 


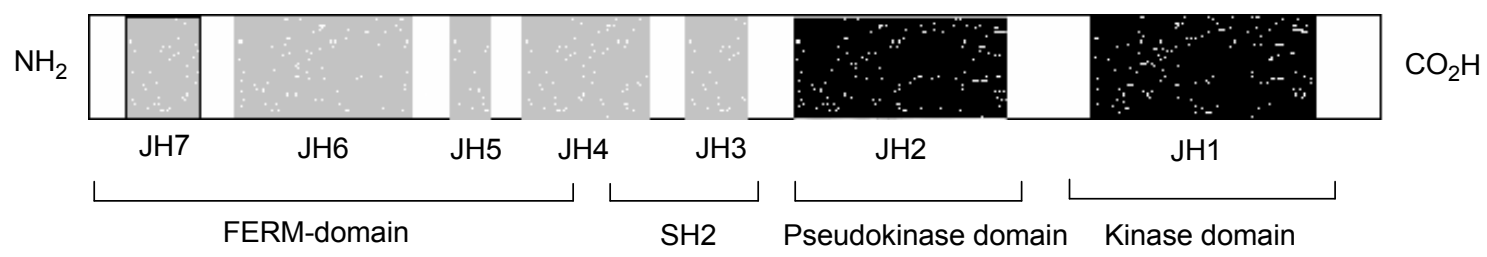

Figure 1: family members of JAKs.

\begin{tabular}{|c|c|c|c|}
\hline Name & Targets & Phase & Company \\
\hline \multicolumn{4}{|l|}{ pan-JAKs inhibitors } \\
\hline Ruxolitinib / INCB-18424, INC-424 & JAK1, JAK2, JAK3 & 2011 Filed & Incyte \\
\hline Tasocitinib / tofacitinib, CP-690550 & JAK1, JAK2, TYK2 & 2012 Filed & Pfizer \\
\hline Baricitinib/ INCB-28050, LY-3009104 & JAK1, JAK2 & Phase III & Incyte \\
\hline GLPG-0634 & JAK1, JAK2 & Phase II & Galapagos \\
\hline CYT-387 & JAK1, JAK2, TYK2 & Phase II & Cytopia \\
\hline AT-9283 & JAK2, JAK3, BCR-ABL & Phase II & Astex \\
\hline INCB-16562 & JAK1, JAK2 & Preclinical & Incyte \\
\hline \multicolumn{4}{|l|}{ Selective JAK2 inhibitors } \\
\hline Pacritinib/ SB-1518 & JAK2/FLT3 & Phase III & S*Bio \\
\hline Lestaurtinib / CEP-701 & JAK2/PDGFR & Phase III & Caephalon \\
\hline AZD-1480 & JAK2 & Phase II & AstraZeneca \\
\hline SAR-302503/ TG-101348 & JAK2/RET & Phase II & Sanofi-Aventis \\
\hline LY-2784544 & JAK2 & Phase II & Lilly \\
\hline BMS-911453 & JAK2 & Phase I & Bristol-Myers Squibb \\
\hline NS-018 & JAK2 & Phase I & Nippon Shinyaku \\
\hline TG02/ SB-1317 & JAK2/CDK, FLT3 & Phase I & S*Bio \\
\hline SB-1578 & JAK2/FLT3 & Phase I & S*Bio \\
\hline XL-019 & JAK2 & $\begin{array}{c}\text { Phase I } \\
\text { (Termination) }\end{array}$ & Exelixis \\
\hline AG-490 & JAK2 & Tools compounds & No company \\
\hline \multicolumn{4}{|l|}{ Selective JAK3 inhibitors } \\
\hline R-348 & JAK3 & Phase I & Rigel \\
\hline VX-509 & JAK3 & Phase I & Vertex \\
\hline NC-1153 & JAK3 & Preclinical & Texas Uni. \\
\hline
\end{tabular}

Table 1: Investigation of JAKs inhibitors.

of haematopoietic cells, as well as many kinds of reactions that are generated by immune and inflammatory processes [9].

JAK3 is a key cell signalling molecule in the immune response, which is specifically distributed in the lymphatic system [10]. In the lymphatic system, interleukin-2 (IL2) can activate JAK3 within a very short period of time. After a period of signal transduction, JAK3 can dephosphorylate and become inactive, so that signals generating quenching facilitate the next round of stimulus signal transmission. Thus, the inhibition of JAK3 activity will prevent side effects caused by damage to other tissues $[11,12]$.

\section{JAKS Inhibitors Overview}

According to the differences between homologous subtypes of JAKs proteins and interaction between inhibitors and JAKs, which occur via contact with the ATP-binding region and the different functions with inhibitors, JAKs inhibitors can be divided into three categories. The first is a pan-JAKs inhibitor, which has a similar degree of inhibition to JAK1, JAK2 and JAK3. The second is the selective inhibitor, which targets JAK2 protein, and is mainly used for antitumour therapy. The third is the selective inhibitor which is targeted to the JAK3 protein, and is most commonly used for anti-inflammatory and immunosuppressive therapy. Since the pairing of JAKs with given cytokine receptors is determined by the chain associated with specific receptor, due to particular cytokines possibly being associated with the pathogenesis of certain diseases, other modulators may cause undesirable side effects. Therefore, it is necessary to develop appropriate inhibitors. Even though the structures of all four JAK family members had been reported, and structure-activity relationships of JAKs are known, it is still a challenge for medicinal chemists to develop JAKs inhibitors because of the similarities between these enzymes. Since the ATP-binding domain consists of 120 amino acids, protein isoforms of JAKs have only 9 different amino acid residues [13]. Therefore, the first generation of non-selective JAKs inhibitors were small molecule compounds. However, with the continuous access to biological protein crystals data, many research institutions developed second generation inhibitors of different subtypes with a broader spectrum, which can inhibit single JAKs proteins with high selectivity $[14,15]$. Currently, the first generation of non-selective inhibitors has been divided in terms of structure into pyrrolopyrimidine compounds (Ruxolitinib, Tasocitinib, INCB-28050), pyrrolopyridine, pyrrolopyrazine etc. Among them, Ruxolitinib from Novartis has been approved for the treatment of myelofibrosis disease, and Pfizer's Tasocitinib has been approved for the treatment of rheumatoid arthritis. The second generation of targeted JAK2 inhibitors includes Lestaurtinib, AZD1480, SB1518, SAR302503, and for JAK3 includes VX509 and R348 (Table 1). The pharmacological effects and clinical application of these compounds are as follows. 


\section{Pan JAK3 Inhibitors}

\section{Ruxolitinib}

Ruxolitinib (INCB18424, INC424) [16] is a JAKs inhibitor which was the first FDA-approved inhibitor for the clinical treatment of myelofibrosis [17]. It was developed by Novartis and U.S. Incyte Biopharmaceutical Company. Ruxilitinib is in clinical phase studies, for indications including other types of cancer (such as lymphoma and pancreatic cancer), polycythaemia vera and plaque psoriasis. How to synthesise this product has not yet be described in the literature. Ruxolitinib, which uses a hinged pyrrolo pyrimidine heterocycle (Figure 2) as a pharmacodynamic substituent, is able to interact with JAKs.

According to the literature, Ruxolitinib is specific for JAK1 and JAK2, as shown using the kinase method. However, JAK1 is involved in other JAKs receptor pairings, including JAK3; therefore, we can adjust the same cytokines as JAK3 signalling. In terms of kinases, the potential advantage of specificity is not obvious. However, in efficacy, the subtle changes are clinically obvious. Ruxolitinib is a Pan JAKs inhibitor. In pre-clinical studies, it has been shown that Ruxolitinib can efficiently inhibit JAK1 $\left(\mathrm{IC}_{50}: 3.3 \mathrm{nM}\right)$, and JAK2 $\left(\mathrm{IC}_{50}: 2.8 \mathrm{nM}\right)$ and weakly inhibit TYK2 $\left(\mathrm{IC}_{50}: 19 \mathrm{nM}\right)$, and JAK3 ( $\left.\mathrm{IC}_{50}: 428 \mathrm{nM}\right)$ [18]. Observed in about $70 \%$ of patients, dysfunction of JAK-STAT signalling is characteristic of some myeloproliferative neoplasms. Mutations in JAK2 caused a number of phenomena. Therefore, the use of JAK2 inhibitors is a viable therapeutic strategy for these patients. Ruxolitinib can be used for the treatment of myeloproliferative neoplasms and has become a promising method for the treatment of bone marrow fibrosis. Although the use of JAK2 inhibitors is compelling in the treatment of bone marrow fibrosis in the presence of the activating mutation JAKv617F, the number of cells expressing V167F alleles would not decrease with the treatment of Ruxolitinib. Cytokine-dependent regulation of JAK1 causes some side effects when using Ruxolitinib. In whole blood assays, it has been shown that Ruxolitinib can inhibit cytokine stimulation by IL-6 (mediated through JAK1) and TPO (mediated through JAK2). In the in vitro experiments, it has been shown that Ruxolitinib can inhibit growth and promote apoptosis in cell lines and primary patient samples carrying JAK2V617F. In mouse models of JAK2V617Fdriven myeloproliferative disease, treatment with ruxolitinib resulted in reduced splenomegaly and improved survival [16]. In addition, in double-blind plaque psoriasis patients, ruxolitinib was used in phase II trials, and patients with topical ruxolitinib cream all had good results for the safety and tolerability of doses, compared with the solvent control groups.

Studies have found that a concentration of Ruxolitinib of less than $1 \mathrm{nmol} / \mathrm{L}$ can inhibit JAK2 and the selectivity to JAK2 is 500 times higher than that for other kinases. Ruxolitinib can inhibit JAK2V627F mutant FDCP and $\mathrm{BaF} / 3$ cell proliferation with an $\mathrm{IC}_{50} 100-130 \mathrm{nmol}$. The inhibitory effect of Ruxolitinib on cell proliferation has a good correlation with the reduction of JAK2 and STAT5 phosphorylation levels of $\mathrm{BaF} / 3$ cells in this model. It has been shown that the inhibition

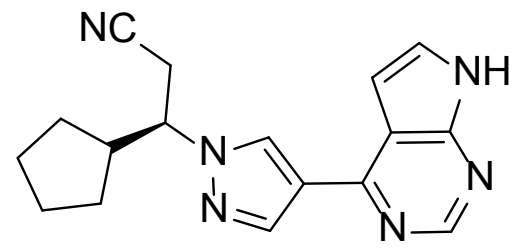

Figure 2: Structure of Ruxolitinib. is mediated by blocking the JAK/STAT channel. By collecting JAK2V617F-positive patients with polycythaemia vera cells for colony formation analysis, we found that Ruxolitinib inhibited cytokinedependent erythroid progenitor cell colony formation $\left(\mathrm{IC}_{50}=67 \mathrm{nmol}\right)$; in normal cells of healthy donors, the colony formation $\mathrm{IC}_{50}$ was greater than $400 \mathrm{nmol} / \mathrm{L}$. At the same time, rat pharmacokinetic experiments showed good bioavailability. In the literature, a study gave SD and Long-Evans rats a single dose of 14C-Ruxolitinib orally. The studies of mass balance and metabolism were conducted in $\mathrm{CByB} 6 \mathrm{~F} 1$ hybrid mice, SD rats and beagle dogs. The route of administration was orally (mouse, rat, dog) or via intravenous injection (rat). The results have shown that Ruxolitinib in SD and Long-Evans rats showed similar tissue distribution and the concentrations in animal gastrointestinal region, bladder, bile, kidney cortex, medulla of kidney, liver, aorta, adrenal, pigment layer and the skin reached a peak within 4 hours after drug administration. In SD rats, except for the aorta, skin and liver, drugs can be completely removed from most tissues. In rats, the results for the oral route were similar to the intravenous administration with regard to the route of elimination and levels. The elimination rates were $92 \%$ and $87 \%$, respectively. Drugs were excreted after 12 hours. Ruxolitinib was also eliminated from dogs very quickly. The amount of drug in the urine and faeces was assessed and found to be $34-36 \%$ and $55-58 \%$. There were small differences between species for Ruxolitinib metabolism, mainly including hydroxylated metabolites, ketones or O-glucuronide substances. In mice and dogs, however, gender differences of Ruxolitinib following serum pharmacokinetics studies were very small. Because the male rats possess specific CYP2C11, CYP2C13, and CYP3A2 enzymes and female rats only possess a specific CYP2C12 enzyme, the male rats produce more data on circulating levels than female rats.

The final approval of the drug was based on two clinical trials regarding safety and efficacy data in 528 patients. All patients in the two trials presented with splenomegaly and ineffective reaction or resistance to existing myelofibrosis drugs or failed bone marrow transplantation. Patients were randomly assigned to receive placebo (sugar pill), Ruxolitinib, or the most effective drugs (such as hydroxyurea or glucocorticoid). The results showed that Ruxolitinib was a more effective treatment for patients whose spleen volume had reduced by more than $35 \%$; that was higher than the other two groups. The data regarding abdominal discomfort, night sweats and bone (or muscle) pain, as well as other symptoms associated with myelofibrosis, reduced by more than $50 \%$ in patients [19]. The most serious adverse reactions to Ruxolitinib include thrombocytopenia, anaemia, fatigue, diarrhoea, difficulty breathing, headaches, dizziness and nausea [19].

\section{Tasocitinib}

Tasocitinib (Tafacitinib, 2) [20] is a JAKs inhibitor for the treatment of moderate to severe rheumatoid arthritis (RA) which was developed by Pfizer. In November 2012, Tasocitinib was approved by the FDA, who stated that it can mainly be applied to patients with RA who have an inadequate response to methotrexate or cannot tolerate the drug. Tasocitinib potently inhibits the enzymatic activity of JAK1 $(3.2 \mathrm{nM})$, JAK2 $(4.1 \mathrm{nM})$ and JAK3 (1.6 nM), with weaker effects on TYK2 (19 $\mathrm{nM})$ [21]. Tasocitinib uses hydrogen bond interactions with the hinge region, thereby making this possible. It can also interact directly with the glycine rich loop (P-loop). In the presence of Tasocitinib, the piperidinemethyl group binds in a lipophilic pocket in the $\mathrm{C}$-terminal lobe at the base of the active site via van der Waals interactions with key residues. Tasocitinib can exquisitely interact with JAKs because of the high degree of shape complementarity of JAKs and Tasocitinib (Figure 3) $[22]$. 


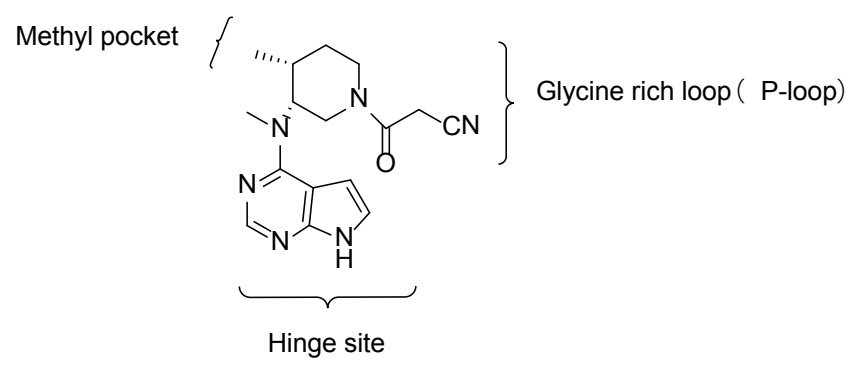

Figure 3: Tasocitinib with JAKs binding sites.

When Pfizer developed the JAKs inhibitors, they obtained the lead compounds CP-35266 (A) with the help of virtual library screening. Medicinal chemists developed new compounds with pharmacophores based on the pyrrolopyrimidine pharmacophoric subunit of CP-352664. The pyrrolopyrimidine ring is assumed to be the hinge-binding element of the molecule and early exploration is based on the relationship between structure and activity around it. It has been found that when they choose to replace this heterocycle or make additional substitutions around the ring, this will generally reduce the potency. A substitution at C-3 is tolerated in JAK3 potency. However, these modifications often resulted in suboptimal metabolic stability, as indicated by human liver microsome incubations and/or diminished cell potency. As a consequence of this initial survey, efforts should be focused on optimisation of the amino head-group in order to achieve program objectives. There are already data showing that these modifications should be put in an $N$-methylcycloalkyl head-group to obtain compound B.

According to the structure-activity relationships, it has been reported that compound libraries are obtained with substituted 2'-position of compound B. The compound with methyl substitution will show good results. Then, using natural products such as synthon can solve the question of two enantiomers of compounds C. Natural terpenoids "carvone" with the desired cyclohexyl can meet the requirements of low cost and stereochemistry. Due to the substituent effects of cyclohexane and stereochemistry, we can use the $(S)$ carvone $(D)$ as raw material; in this way, we found that all of the derivatives obtained were all-cis configuration. Compound E, using the isopropenyl group as a synthetic handle, will guide the compound to combine with the large pocket. As the characteristic properties of the drug, it is necessary to reduce the complexity and increase the stereochemistry of new analogues by introducing the corresponding substituents such as piperidine to support compound $\mathrm{F}$. The piperidine also facilitated synthetic access to this active site pocket, which is now known to project towards the glycine rich loop (P-loop) of the kinases. Therefore, it is crucial to avoid unnecessary lipophilic side chains and reduce the molecular weight. Finally, the cyanoacetamide side chain of tofacitinib was found to possess the best attributes for JAKs (Figure 4) [22]. According to the literature, there are more than 1000 synthetic analogues of the original lead molecule CP-352664.

The Tasoitinib molecule comes from compound 3 by multiple structural optimisations. At the beginning, it was found that Tasoitinib has a potent inhibitory effect on JAK3 (minimum inhibitory concentration of $1 \mathrm{nmol} \bullet \mathrm{L}^{-1}$ ), but the inhibition of JAK2 and JAK1 was $1 \%$ and $5 \%$ of JAK3, respectively. CP-690550 is considered a specific inhibitor of JAK3. Recombinant separate kinases and biochemical experiments have shown that Tasoitinib is a non-selective JAKs inhibitor. The difference in Tasoitinib's inhibitory activity to JAKs is only 4 to 10 times $[23,24]$.

Preclinical animal studies have shown that Tosoitinib can inhibit the joint tissues of rat models, producing inflammatory mediators and STAT1-dependent genes. It can improve the condition of the rat markedly. For rheumatoid arthritis (RA), after given the remitting anti-rheumatic drugs (DMARDs) and finding them to be an ineffective treatment for RA, the patients were given Tasoitinib. In a phase II study, it has been shown that the level reaching ACR20 (Tender and swollen joint count reduced by at least $20 \%$, and 3 of the other 5 RA severity indexes improved by at least $20 \%$ ) was $67 \%$ among the RA patients who only take Tasoitinib compared with $25 \%$ in the placebo group. The rate of ACR2 0 was $59 \%$ in the group being treated with Tasocitinib and methotrexate, but only $35 \%$ in the group receiving methotrexate [25]. In addition, In addition, in a 12-month phase III clinical trial, 717 patients with moderate to severe RA using methotrexate, were also randomly given Tasocitinib (5mg and $10 \mathrm{mg}$,bid, po), adalimumab (40mg, one Monday times, sc) or placebo. Three months later, the results showed

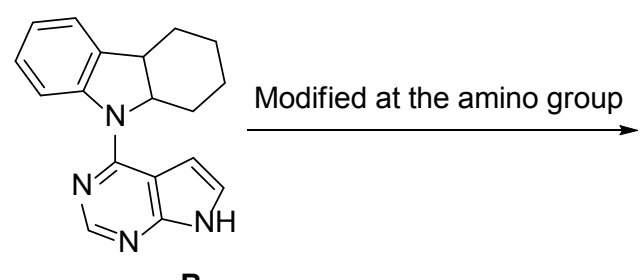

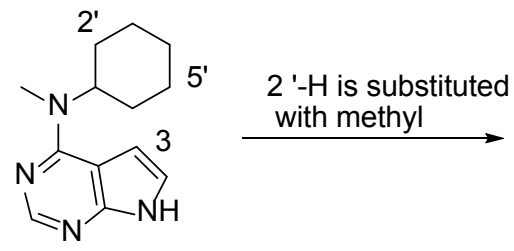

C<smiles>CC1CCC(C)C(N(C)c2ncnc3[nH]ccc23)C1</smiles>

D<smiles>C=C(C)C1CC=C(C)C(=O)C1</smiles><smiles>C=C(C)[C@H]1CC[C@H](C)CC1</smiles>

$\mathbf{F}$

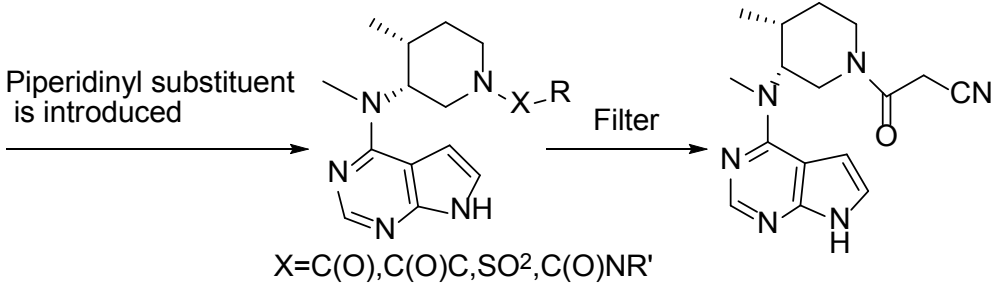

G

Figure 4: Structure of compound 2-8. 
that the body function of patients improved significantly in the two groups of Tasocitinib with HAQDI (Health Questionnaire Disease Index). Six months later, the rate of ACR20 in the four groups was $511.5 \%, 52.5 \%, 47.2 \%, 28.3 \%$ respectively $[25,26]$. Common adverse reactions to Tasocitinib include elevated blood cholesterol levels, neutropenia, upper respiratory tract infection, headache and diarrhoea $[27,28]$.

Tasocitinib is the treatment of choice for moderate to severe rheumatoid arthritis; also, as reported in the literature, it has a certain effect on moderate to severe ulcerative colitis. For ulcerative colitis (UC), the study of Tasocitinib is in phase II; the double-blinded, placebo-controlled study included patients with moderately to severely active UC. Patients were given tofacitinib at doses of $0.5,3,10$, or 15 mg or placebo twice daily for 8 weeks. Clinical response occurred in $32 \%, 48 \%, 61 \%$, and $78 \%$ of patients, respectively, compared with $42 \%$ in the placebo group. Clinical remission occurred in $13 \%, 33 \%, 48 \%$, and $41 \%$ of patients, respectively, compared with $10 \%$ in the placebo group. These data show that patients with moderately to severely active UC receiving tofacitinib were more likely to have a clinical response or remission than those receiving placebo. For the treatment of UC, the study of Tofacitinib has entered phase III [29].

\section{Baricitinib}

Baricitinib (INCB-28050, LY3009104,9) [30], which was codeveloped by Incyte and Lilly, can be a treatment for rheumatoid arthritis, inflammatory diseases and cancer. Baricitinib, which uses a hinged pyrrolopyrimidine heterocycle as a pharmacodynamic substituent, interacts with JAKs [31]. It is a hetero aryl group substituted pyrrolopyrimidine compound (Figure 5). Its synthesis method has not yet been reported in the literature [22].

The in vitro experiments have shown that it has a good oral availability of JAK1/JAK2 inhibitor ( IC $_{50}$, JAK1: 59nM, JAK2: $57 \mathrm{nM}$ ), with little activity against JAK3. In the experiments, Baricitinib demonstrated good tolerability and appropriate efficacy [31]. In November 2010, Incyte recruited 100 patients with rheumatoid arthritis who participated in a six-month double-blind, placebo-controlled, single and multiple ascending measurement study, progressing to a phase II clinical trial [32]. Patients were administered doses of $4 \mathrm{mg}$, $7 \mathrm{mg}$ and $10 \mathrm{mg}$ once per day for 12 weeks. The studies showed that Baricitinib can significantly improve the ARC20 (59\% ARC20, 35\% ARC50, and 16\% ARC70). In the same conditions, patients were given placebo. It has been found that the improvements of ARC20, ARC50, and ARC70 are $32 \%, 13 \%$ and 3\%, respectively. The main side effects of Baricitinib such as mild or moderate decreases in haemoglobin are similar to the performance of placebo. The phase III studies of Baricitinib are currently underway. Rodents with arthritis have been tested using Baricitinib and Ruxolitinib. The results have shown that both Baricitinib and Ruxolitinib have appropriate efficacy. By increasing the dose of Baricitinib in rat experiments, the histological and radiographic signs of the disease in rats will increase. Baricitinib is a drug that deserves development and research. A comparison of Tasocitinib, Ruxolitinib, and Baricitinib is shown in Table 2. As shown in Table 2, the pyrrolopyrimidine group is the most important pharmacophore for JAKs inhibitors.

\section{GLPG-0634}

GLPG-0634 is an oral JAKs inhibitor for the treatment of rheumatoid arthritis, which was developed by Galapagos. The structure of GLPG-0634 has not yet been announced; however, it is known to belong to the class of triazolopyridine. The structure of a GLPG-0634 analogue is shown in Figure 6 [33].

The studies of GLPG-0634 are now in Phase II. Preclinical studies have shown that GLPG-0634 is a JAK1 and JAK2 inhibitor (JAK1: $10 \mathrm{nM}$, JAK2: $28 \mathrm{nM}$, JAK3: $810 \mathrm{nM}$ ). GLPG-0634 shows excellent therapeutic activity in vitro and in vivo studies of rheumatoid disease models [34]. The Galapagos Company is focusing on the research

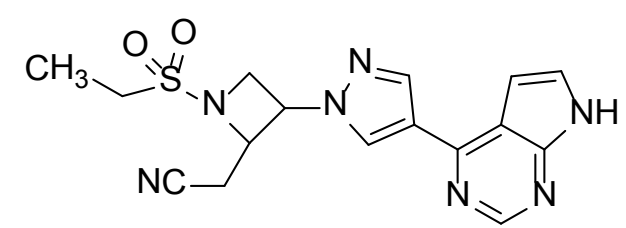

Figure 5: The structure of Baricitinib.

\begin{tabular}{|c|c|c|c|c|c|c|}
\hline & \multicolumn{4}{|c|}{ Inhibition of JAKs IC ${ }_{50}(\mathrm{nM})$} & \multirow{2}{*}{ Structure } & \multirow{2}{*}{ Pharmacophore } \\
\hline & JAK1 & JAK2 & JAK3 & TYK2 & & \\
\hline Tasocitinib & 3.3 & 2.8 & 19 & 428 & & pyrrolopyrimidine \\
\hline Ruxolitinib & 3.2 & 4.1 & 1.6 & 19 & & pyrrolopyrimidine \\
\hline Baricitinib & 59 & 57 & - & - & & pyrrolopyrimidine \\
\hline
\end{tabular}

Table 2: Comparison of Tasocitinib, Ruxolitinib, Baricitinib. 
<smiles>N#Cc1ccc(COc2ccc(-c3cccc4nc(NC(=O)C5CC5)nn34)cc2)cn1</smiles>

Figure 6: Structure of the GLPG-0634 analogue.

and development of GLPG-0634, because of its high inhibitory effect on JAK-1/2, meaning that it can improve the safety of treatment of rheumatic diseases and reduce side effects such as anaemia. Compared with JAK3 inhibitors, it has better outcomes. Phase I studies were carried out in Belgium in May 2010, including double-blind, placebocontrolled, single and multiple dose escalation study programmes $(\mathrm{N}=$ 40); this research ended in June, 2011. The following phase II studies were conducted in Eastern Europe [32]. A total of 36 patients with RA

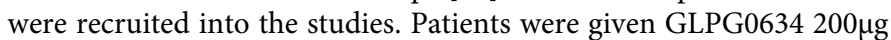
(qd), $100 \mu \mathrm{g}$ (bid) or placebo. As a result, both the groups of GLPG0634 reached the primary endpoint of the expected indicators. Galapagos Company Development Senior Vice Piet WigeRinck pointed out that although the time of the trial was short, GLPG-0634 had already shown more effectiveness than the previously reported initial drugs for the treatment of RA. In the future, if GLPG-0634 can maintain its high efficiency and good security in long-term clinical studies, it may become a "heavyweight" therapeutic agent for RA and other inflammatory diseases.

\section{CYT-387}

CYT-387 is a drug developed by Cytopia for the treatment of idiopathic myelofibrosis (Figure 7). It is an amino pyrimidine compound. CYT-387 can strongly inhibit the JAKs kinase (JAK1:11nm, JAK2:18nm, TYK:17nm) [35,36]. Currently it is still in l phase II study.

Preclinical studies have shown that this drug has higher activity in the cell model, the main sample of patients and animal models. In 2010, it completed phase I/II clinical trials and the results of these clinical trials were updated in 2012. Experiments have shown that, in the 36 patients with high risk primary myelofibrosis, polycythaemia vera myelofibrosis and primary thrombocytosis after bone marrow fibrosis, there were no grade 4 non-haematological adverse reactions, and there were grade 2 cases with 3 non-haematological adverse reactions, 2 cases with elevated alkaline phosphatase, 2 cases with headache, 1 case of serum lipase, and 1 case with prolonged QTc; a total of 36\% (13 cases) emerged from the first effective limiting including Light-headedness and hypotension, $22 \%$ ( 8 cases) had grade $3 / 4$ thrombocytopenia and one case reported grade three anaemia with sudden onset. In effectiveness, the symptoms of anaemia improved the response rate to $63 \%$; overall, $37 \%$ of patients had splenic volume decreased by at least $50 \%$. The conclusion is that the CYT-387 in the treatment of bone marrow fibrosis has a significant effect and good tolerance. The main side effects are headache and hypertension. At present, the company is carrying out an phase II clinical study with an increased sample set $[37,38]$.

\section{Selective JAK3 Inhibitors}

\section{Pacritinib}

Pacritinib (SB-1518, 12) is an oral active JAK2 $\left(\mathrm{IC}_{50}: 22 \mathrm{nM}\right)$ inhibitor developed by $\mathrm{S}^{\star} \mathrm{BIO}$ company. Pacritinib (Figure 8 ) belongs to the class of macrocyclic aminopyrimidine compounds [39].

Pacritinib can be the treatment of primary myelofibrosis, polycythaemia vera after bone marrow fibrosis and idiopathic thrombocythaemia myelofibrosis. SB-1518 has received orphan drug status in Europe and the United States for the treatment of these diseases [40]. The data of phase I and II clinical trials have shown that Pacritinib has good activity and tolerability in the treatment of myelofibrosis. Currently, a double-blind and placebo-controlled Phase III study is being launched in myelofibrosis patients with splenomegaly. The primary purpose of these studies is to display significant differences between Pacritinib and placebo in reducing the size of the spleen [41].

\section{AZD-1480}

AZD-1480 is a small molecule JAK2 inhibitor developed by AstraZeneca for the treatment of bone marrow fibrosis and hyperplasia $[42,43]$. It belongs to the group of amino pyrimidine derivatives (Figure 9).

The in vitro studies have shown that AZD-1480 is a highly efficient inhibitor of JAK2 ( $\left.\mathrm{IC}_{50}: 0.26 \mathrm{nM}\right)$. Clinical findings of phase I studies in 80 patients with primary myelofibrosis indicated that AZD-1480 has good tolerance. In preclinical studies, experiments on mice with cancer have been conducted in vivo. The data have shown that AZD1480 can significantly inhibit the growth of IL-6, and can effectively<smiles>C#CCNC(=O)c1ccc(-c2ccnc(Nc3ccc(N4CCOCC4)cc3)n2)cc1</smiles>

Figure 7: Chemical structure of CYT-387.<smiles>C/C=C\COC/C=C\COCc1cccc(-c2ccnc(Nc3ccc(OCCN4CCCC4)c(COCCN4CCCC4)c3)n2)c1</smiles>

Figure 8: Structure of Pacritinib.<smiles>Cc1cc(Nc2nc(N[C@H](C)c3ncc(F)cn3)ncc2Cl)n[nH]1</smiles>

Figure 9: Structure of AZD-1480. 
inhibit the STAT3 signalling pathway in solid tumours. It has been confirmed that in human tumour cells, JAK2 plays an important role in basal regulation and in the activation of cytokine-induced STAT3. If JAK2 activity can be inhibited, tumorigenesis can be suppressed as well. Other selective JAK2 inhibitors are listed in Table 3.

\section{Selective JAK3 Inhibitors}

\section{R-348}

R-348 is a JAK3 inhibitor which was developed by the Rigel pharmaceutical company in cooperation with Stanford University [51]. We cannot determine its structure from the literature. R-348 can treat autoimmune diseases, including psoriasis, rheumatoid arthritis (RA) and graft rejection. Currently, R-348 is in phase I research for keratoconjunctivitis sicca. Preclinical test results have shown that R-348 can effectively prevent the development of OAD and reduce the infiltration of mononuclear cells. R-348 also has a great impact on reducing the level of IgG antibodies. In a heteRoRopic heart transplantation model test, R-348 was found to effectively prevent acute allograft rejection and inhibit the systemic immune response, whilst prolonging the survival rate of transplantation organisms.

\section{VX-509}

VX-509 is a novel oral selective JAK3 inhibitor developed by VeRtex for the treatment of rheumatoid arthritis. We cannot determine its structure from the literature. VX-509 strongly inhibits JAK3; the strength of this inhibition is 25-100 times that of other kinases. Researchers have found that the largest dose of the product is $150 \mathrm{mg} /$ day in a phase I 14 day clinical study and phase II study of 200 rheumatoid arthritis patients. Patients who used methotrexate in the early stages can achieve the basic requirements of ACR20 and improve the DAS28 index by using VX-509 after 12 weeks of treatment. The results were similar to those for Tasocitinib alone [52]. The comparison of several JAKs inhibitors for the treatment of rheumatoid arthritis is shown in Table 4. Because the target of VX-509 is different to other inhibitors, VX-509 is expected to show superior performance for the treatment of rheumatoid arthritis. A larger clinical trial is underway.

Currently, JAK3 inhibitors are being investigated by medicinal chemists and immunologists [53]. In addition to the JAK3 inhibitors above, there are a variety of such inhibitors reported in the literature, such as 2-naphthyl ketone compounds, quinoline derivatives, and two methyl xanthine compounds. However, because these compounds do not have high selectivity for JAK3 and large side effects, we have not reported them. New JAK3 selective inhibitors will play an important role in organ transplantation. JAK3 inhibitors have important significance in organ transplantation and the treatment of autoimmune diseases.

\begin{tabular}{|c|c|c|c|c|}
\hline Name & Targets & Structure & Type of Structure /Function /Side effects & Company /Phase \\
\hline $\begin{array}{c}\text { SAR-302503/ TG- } \\
101348\end{array}$ & JAK2/RET & & $\begin{array}{l}\text { Aminopyrimidines; } \\
\text { Drug of Ph chromosome-negative } \\
\text { myeloproliferative neoplasms [44,45]; Nausea, } \\
\text { diarrhoea, anaemia [46] }\end{array}$ & $\begin{array}{l}\text { Sanofi- } \\
\text { Aventis } \\
\text { Phase II }\end{array}$ \\
\hline LY-2784544 & JAK2 & & $\begin{array}{l}\text { Imidazolepyrazole; } \\
\text { Polycythaemia vera, essential- } \\
\text { Thrombocythaemia; } \\
\text { Tumour lysis syndrome [47] }\end{array}$ & $\begin{array}{c}\text { Lilly } \\
\text { Phase II }\end{array}$ \\
\hline BMS-911453 & JAK2 & & $\begin{array}{l}\text { Pyrrole imidazopyridine; } \\
\text { Inhibited CD34 + from bone marrow fibrosis; } \\
\text { Not determined [48] }\end{array}$ & $\begin{array}{l}\text { BRistol- } \\
\text { MyeRsSquibb } \\
\text { Phase I }\end{array}$ \\
\hline $\mathrm{XL}-019$ & JAK2 & & $\begin{array}{l}\text { Pyrrole amides; } \\
\text { Myeloproliferative disorders [49]; } \\
\text { Phenomenon of central and peripheral } \\
\text { neuropathy }\end{array}$ & $\begin{array}{l}\text { Exelixis } \\
\text { Phasel } \\
\text { (termination) }\end{array}$ \\
\hline AG-490 & JAK2 & & $\begin{array}{l}\text { Styrene amine; } \\
\text { Specific JAK2 antagonist [50]; } \\
\text { Tools compound }\end{array}$ & $\begin{array}{l}\text { No reports of } \\
\text { clinical studies }\end{array}$ \\
\hline
\end{tabular}

Table 3: Presentation of other selective JAK2 inhibitors. 
Citation: Jiang JJJ, Wang XY, Zhang Y, Jin Y, Lin J (2014) Advances in the Inhibitors of Janus Kinase. Med chem 4: 540-548. doi:10.4172/21610444.1000192

\begin{tabular}{|c|c|c|}
\hline Name & Targets & Phase \\
\hline Tofacitinib & JAK3>JAK1>JAK2 & Filed \\
\hline INCB28050 & JAK1=JAK2 & Phase III \\
\hline GLPG0634 & JAK1 & Phase II \\
\hline VX-509 & JAK3 & Phase II \\
\hline
\end{tabular}

Table 4: Compare of several JAKs inhibitors' treatment of rheumatoid arthritis.

\section{Conclusion}

About twenty years have passed since JAKs inhibitors were first identified. Now, small molecular JAKs inhibitors have been shown to be the most promising drugs for the treatment of immune-mediated diseases. The first generation of non-selective targeting drugs of JAKs have shown clinical efficacy against a broad spectrum of autoimmune diseases. However, the JAKs drugs that have appeared on the market recently also show some clinical side effects; what is worse, these side effects may limit their clinical application. In terms of the clinical side effects, the second generation of selective JAK2 inhibitors (such as JAK2 or JAK selective inhibitor) has been exploited to reduce these side effects. These JAK2 inhibitors can not only improve the selectivity of JAK, but can also, at the same time, increase the security of its clinical application. However, the final effects of their clinical application need further trial data.

\section{Acknowledgment}

This work was supported by the Program for Changjiang Scholars and Innovative Research Team in University (No. IRT13095), the National Natural Science Foundation of China (Nos. 21262043, 20902079, 81160384, 21262042 and U1202221). The authors thank the High Performance Computing Center at Yunnan University for use of the high performance computing platform.

\section{References}

1. Daub H, Specht K, Ullrich A (2004) Strategies to overcome resistance to targeted protein kinase inhibitors. Nat Rev Drug Discov 3: 1001-1010.

2. Aggarwal BB, Kunnumakkara AB, Harikumar KB, Gupta SR, Tharakan ST, et al. (2009) Signal transducer and activator of transcription-3, inflammation, and cancer: how intimate is the relationship?. Ann N Y Acad Sci 1171: 59-76.

3. Tam CS, Verstovsek S (2013) Investigational Janus kinase inhibitors. Expert Opin Investig Drugs 22: 687-699.

4. Alicea-VelÃ $i z q u e z ~ N L$, Boggon TJ (2011) The use of structural biology in Janus kinase targeted drug discovery. Curr Drug Targets 12: 546-555.

5. Behrmann I, Smyczek T, Heinrich PC (2004) Janus kinase (Jak) subcellular localization revisited: the exclusive membrane localization of endogenous Janus kinase 1 by cytokine receptor interaction uncovers the Jak. receptor complex to be equivalent to a receptor tyrosine kinase. J Biol Chem 279: 35486-35493.

6. Remy I, Wilson IA, Michnick SW (1999) Erythropoietin receptor activation by a ligand-induced conformation change. Science 283: 990-993.

7. Rane SG, Reddy EP (2000) Janus kinases: components of multiple signaling pathways. Oncogene 19: 5662-5679.

8. Flex E, Petrangeli V, Stella L, Chiaretti S, Hornakova T, et al. (2008) Somatically acquired JAK1 mutations in adult acute lymphoblastic leukemia. J Exp Med 205: 751-758.

9. Zhang W, Chen PL, Du L (2009) Research progress of JAK2 structure and its function. Nat Sci J Hainan Univ. (natural science edition) 27: 87-90.

10. Sohn SJ, Forbush KA, Nguyen N, Witthuhn B, Nosaka T, et al. (1998) Requirement for Jak3 in mature $\mathrm{T}$ cells: its role in regulation of $\mathrm{T}$ cell homeostasis. J Immunol 160: 2130-2138.

11. Vincenti $F$ (2003) Immunosuppression minimization: current and future trends in transplant immunosuppression. J Am Soc Nephrol 14: 1940-1948.

12. He XH, Zhang ZB, Li S (2005) Research progress of tyrosine kinase JAK3 inhibitor. Chin J Med Chem15: 373-377.

13. Wilks AF (2008) The JAK kinases: not just another kinase drug discovery target. Semin Cell Dev Biol 19: 319-328.

14. Lucet IS, Fantino E, Styles M, Bamert R, Patel O, et al. (2006) The structural basis of Janus kinase 2 inhibition by a potent and specific pan-Janus kinase inhibitor. Blood 107: 176-183.

15. Chrencik JE, Patny A, Leung IK, Korniski B, Emmons TL, et al. (2010) Structural and thermodynamic characterization of the TYK2 and JAK3 kinase domains in complex with CP-690550 and CMP-6. J Mol Biol 400: 413-433.

16. Quint $\tilde{A}_{j}$ S-Cardama A, Vaddi K, Liu P, Manshouri T, Li J, et al. (2010) Preclinica characterization of the selective JAK1/2 inhibitor INCB018424: therapeutic implications for the treatment of myeloproliferative neoplasms. Blood 115: 3109-3117.

17. Mesa RA, Yasothan U, Kirkpatrick P (2012) Ruxolitinib. Nat Rev Drug Discov 11: 103-104.

18. Cervantes F, Dupriez B, Pereira A, Passamonti F, Reilly JT, et al. (2009) New prognostic scoring system for primary myelofibrosis based on a study of the International Working Group for Myelofibrosis Research and Treatment. Blood 113: $2895-2901$.

19. Verstovsek S, Kantarjian H, Mesa RA, Pardanani AD, Cortes-Franco J, et al. (2010) Safety and efficacy of INCB018424, a JAK1 and JAK2 inhibitor, in myelofibrosis. N Engl J Med 363: 1117-1127.

20. Flanagan ME, Blumenkopf TA, Brissette WH, Brown MF, Casavant JM, et al (2010) Discovery of CP-690,550: a potent and selective Janus kinase (JAK) inhibitor for the treatment of autoimmune diseases and organ transplant rejection. J Med Chem 53: 8468-8484.

21. Changelian PS, Flanagan ME, Ball DJ, Kent CR, Magnuson KS, et al. (2003) Prevention of organ allograft rejection by a specific Janus kinase 3 inhibitor. Science 302: 875-878.

22. Clark JD, Flanagan ME, Telliez JB (2014) Discovery and Development of Janus Kinase (JAK) Inhibitors for Inflammatory Diseases. J Med Chem 57: 5023-5038.

23. Williams NK, Bamert RS, Patel O, Wang C, Walden PM, et al. (2009) Dissecting specificity in the Janus kinases: the structures of JAK-specific inhibitors complexed to the JAK1 and JAK2 protein tyrosine kinase domains. J Mol Biol 387: $219-232$

24. Karaman MW, Herrgard S, Treiber DK, Gallant P, Atteridge CE, et al. (2008) A quantitative analysis of kinase inhibitor selectivity. Nat Biotechnol 26: 127-132.

25. Kudlacz E, Conklyn M, Andresen C, Whitney-Pickett C, Changelian P (2008) The JAK-3 inhibitor CP-690550 is a potent anti-inflammatory agent in a murine model of pulmonary eosinophilia. Eur J Pharmacol 582: 154-161.

26. Coombs JH, Bloom BJ, Breedveld FC (2010) Improved pain, physical functioning and health status in patients with rheumatoid arthritis treated with CP-690,550, an orally active Janus kinase (JAK) inhibitor: results from a randomised, double-blind, placebo-controlled trial. Ann Rheum Dis 9: 413-416.

27. Wojciechowski D, Vincenti $F$ (2011) Targeting JAK3 in kidney transplantation: current status and future options. Curr Opin Organ Transplant 16: 614-619.

28. Busque S, Leventhal J, Brennan DC, Steinberg S, Klintmalm G, et al. (2009) Calcineurin-inhibitor-free immunosuppression based on the JAK inhibitor CP690,550: a pilot study in de novo kidney allograft recipients. Am J Transplant 9: 1936-1945

29. Sandborn WJ, Ghosh S, Panes J, Vranic I, Su C, et al. (2012) Tofacitinib, an oral Janus kinase inhibitor, inactive ulcerative colitis. New Engl J Med 367: 616-624.

30. Fridman JS, Scherle PA, Collins R, Burn T, Neilan CL, et al. (2011) Preclinical evaluation of local JAK1 and JAK2 inhibition in cutaneous inflammation. J Invest Dermatol 131: 1838-1844.

31. Fridman JS, Scherle PA, Collins R, Burn TC, Li Y, et al. (2010) Selective inhibition of JAK1 and JAK2 is efficacious in rodent models of arthritis: preclinical characterization of INCB028050. J Immunol 184: 5298-5307.

32. Kontzias A, Kotlyar A, Laurence A, Changelian P, O'Shea JJ (2012) Jakinibs: a new class of kinase inhibitors in cancer and autoimmune disease. Curr Opin Pharmacol 12: 464-470.

33. Manette CJM, Blank J (2009) New compounds for the treatment of degenerative and inflammatory disease. WO200980129124.7.

34. Fan M (2012) GLPG0634 for the treatment of rheumatoid arthritis in phase II trials gets positive results. Prog Pharm Sci 36: 192-193.

35. Pardanani A, Lasho T, Smith G, Burns CJ, Fantino E, et al. (2009) CYT387, a selective JAK1/JAK2 inhibitor: in vitro assessment of kinase selectivity and preclinical studies using cell lines and primary cells from polycythemia vera patients. Leukemia 23: 1441-1445. 
Citation: Jiang JJJ, Wang XY, Zhang Y, Jin Y, Lin J (2014) Advances in the Inhibitors of Janus Kinase. Med chem 4: 540-548. doi:10.4172/21610444.1000192

36. Tyner JW, Bumm TG, Deininger J, Wood L, Aichberger KJ, et al. (2010) CYT387, a novel JAK2 inhibitor, induces hematologic responses and normalizes inflammatory cytokines in murine myeloproliferative neoplasms. Blood 115: 5232-5240

37. Pardanani A, George G, Lasho T (2010) A phase I/II study of CYT387, an oral JAK-1/2 inhibitor, in myelofibrosis: significant response rates in anemia, splenomegaly, and constitutional symptoms. ASH Annl Meet Abstr 116: 460.

38. Pardanani A, Gupta GJ (2011) An expanded multicenter phase I/II study of CYT387, a JAK- $1 / 2$ inhibitor for the treatment of myelofibrosis. ASH Annl Mee Abstr 20, 118, 3849.

39. Hart S, Goh KC, Novotny-Diermayr V, Hu CY, Hentze H, et al. (2011) SB1518, a novel macrocyclic pyrimidine-based JAK2 inhibitor for the treatment of myeloid and lymphoid malignancies. Leukemia 25: 1751-1759.

40. William AD, Lee AC, Blanchard S, Poulsen A, Teo EL, et al. (2011) Discovery of the macrocycle 11-(2-pyrrolidin-1-yl-ethoxy)-14,19-dioxa-5,7,26-triaza-tetracyclo[19.3.1.1(2,6).1(8,12)] heptacosa-1(25),2(26),3,5,8,10,12(27), 16,2,23decaene (SB1518), a potent Janus kinase 2/fms-like tyrosine kinase-3 (JAK2/ FLT3) inhibitor for the treatment of myelofibrosis and lymphoma. J Med Chem 54: 4638-4658.

41. Komrokji RS, Wadleigh M, Seymour JF () Results of a phase II study of Pacritinib (sb1518), a novel oral jak2 inhibitor, in patients with primaRy, postpolycythemia vera, and post-essential thrombocythemia myelofibrosis. ASH Annl Meet Abstr 20, 118, 282.

42. Hedvat M, Huszar D, Herrmann A, Gozgit JM, Schroeder A, et al. (2009) The JAK2 inhibitor AZD1480 potently blocks Stat3 signaling and oncogenesis in solid tumors. Cancer Cell 16: 487-497.

43. Couto JP, Almeida A, Daly L, Sobrinho-SimÃues M, Bromberg JF, et al. (2012) AZD1480 blocks growth and tumorigenesis of RET- activated thyroid cancer cell lines. PLoS One 7: e46869.

44. Lasho TL, Tefferi A, Hood JD, Verstovsek S, Gilliland DG, et al. (2008) TG101348, a JAK2-selective antagonist, inhibits primary hematopoietic cells derived from myeloproliferative disorder patients with JAK2V617F, MPLW515K or JAK2 exon 12 mutations as well as mutation negative patients. Leukemia 22: $1790-1792$.

45. Wernig G, Kharas MG, Okabe R, Moore SA, Leeman DS, et al. (2008) Efficacy of TG101348, a selective JAK2 inhibitor, in treatment of a murine model of JAK2V617F-induced polycythemia vera. Cancer Cell 13: 311-320.

46. Pardanani A, Gotlib JR, Jamieson C, Cortes JE, Talpaz M, et al. (2011) Safety and efficacy of TG101348, a selective JAK2 inhibitor, in myelofibrosis. J Clin Oncol 29: 789-796.

47. Ma L, Zhao B, Walgren R (2010) Efficacy of LY2784544, a Small Molecule Inhibitor Selective forR Mutant JAK2 Kinase, In JAK2 V617F-Induced Hematologic Malignancy Models. ASH Annl Meet Abstr 116: 4087.

48. Verstovsek S, Mesa RA, Rhoades SK (2011) Phase I Study of the JAK2 V617F Inhibitor, LY2784544, in Patients with Myelofibrosis (MF), Polycythemia Vera (PV), and Essential Thrombocythemia (ET). Annl Meet Abstr 118: 2814.

49. Verstovsek S, Pardanani A, Shah N (2007) A phase I study of XL019, a selective JAK2 inhibitor, in patients with primary myelofibrosis and post-polycythemia vera/essential thrombocythemia myelofibrosis. Annl Meet Abstr 110: 553.

50. Meydan N, Grunberger T, Dadi H, Shahar M, Arpaia E, et al. (1996) Inhibition of acute lymphoblastic leukaemia by a Jak-2 inhibitor. Nature 379: 645-648.

51. Deuse T, Velotta JB, Hoyt G, Govaert JA, Taylor V, et al. (2008) Novel immunosuppression: R348, a JAK3- and Syk-inhibitor attenuates acute cardiac allograft rejection. Transplantation 85: 885-892.

52. Hoock T, Hogan J, Mahajan S (2011) Presentation 1136. American College of Rheumatology Annual Scientific Meeting. Abstract: Arthritisrheum. Annl. Meet Abstr 20,63, 1136.

53. Behbod F, Erwin-Cohen RA, Wang ME (2001) Concomitant inhibition of Janus kinase 3 and calcineurin-dependent signaling pathways synergistically pRolongs the survival of rat heart allografts. J. Immunol 166: 3724-3732. 